\title{
Two mechanisms in the stimulus suffix effect
}

\author{
JOHN MORTON \\ Medical Research Council, Applied Psychology Unit, Cambridge, England CB2 2EF
}

\begin{abstract}
The stimulus suffix is a redundant item presented immediately after a stimulus list. Its effect is the selective impairment of recall of the final items in a serially recalled, auditorily presented list of unconnected items. Two experiments indicate that there was no difference between the effects of suffixes .5 and $1.0 \mathrm{sec}$ after the end of a digit list presented at a rate of one digit/sec. This suggests that the effect of the suffix, in this case the vowel sound "ah," is not a simple function of its time of arrival after the final digit, as has been thought. The possibility of more complex factors was supported in a further experiment which showed a slight reduction in the size of the suffix effect by repeating the suffix three times.
\end{abstract}

The stimulus suffix is a redundant item which occurs immediately after an acoustically presented list of items which have to be recalled serially (Crowder \& Morton, 1969; Morton, 1970; Morton, Crowder, \& Prussin, 1971). It has a selective effect on the recall of the last few items in the list, the size of the effect being sensitive to acoustic factors such as the relative spatial location of the pitch of the stimuli and suffix but insensitive to semantic variables. Crowder and Morton (1969) assigned responsibility for this effect to what they called precategorical acoustic storage (PAS). It is supposed that acoustically presented information remains available in PAS for some time after presentation irrespective of whether or not this material has been recognized at the time of presentation. Any further speech stimulus is supposed to have the effect of erasing previous information from PAS. Thus, by the time a list of items has been presented, the only information remaining in PAS would be that resulting from the last item or two. It is a matter of conjecture as to how this information is utilized in the course of recall, but the current best bet is that the information is passively transferred from PAS to some longer term store (Routh, 1971). Whatever the actual process, Crowder (1969) has indicated that it must take place within about $2 \mathrm{sec}$ of the presentation of the last item. In his experiment subjects were presented with a list of nine digits at a rate of two digits/sec. The lists were followed by a redundant suffix, "zero," at $.5,2,5$, or $10 \mathrm{sec}$ after the last item. The condition with a .5 -sec suffix showed the usual large decrement on the last item. There were no differences among the 2,5, and 10-sec suffix conditions, in all of which there was no suffix effect. The conclusion was that the process which utilized the information in PAS had been completed within $2 \mathrm{sec}$, by which time this information was secure. In a further experiment, Crowder (1971, Experiment 1) showed that a suffix

I am grateful to Byron Morgan for providing a program for the Wilcoxon tests and to Keith Taylor for his kind assistance with the data processing. The assistance of Susan Chambers and Pennie Ottley in running the experiments is gratefully acknowledged. had progressively less effect as it was presented at .5 , $.6, .8,1.0$, and $1.5 \mathrm{sec}$ after the last item. This refinement could again be interpreted as showing that the utilization of the PAS information takes time.

In both of the above experiments, the stimulus items were presented at a rate of two items/sec. This meant that the shortest time interval used was the same as the interitem time, and it is thus not possible to decide between interpretations based on the time between the last item and the suffix and interpretations based on the extent to which the suffix occurs in rhythm with the stimuli. In the latter case "time of arrival" might be considered to be a property of the stimuli and suffix which can be used by some attention mechanism. A suffix which occurs arhythmically with respect to the stimulus list might then have less effect in the same way that a suffix in the opposite ear to or in a different voice from the stimulus items has a reduced effect (Morton et al., 1971). Crowder (1971, Experiment 3) tested the time and rhythm hypothesis in an experiment in which he covaried interitem times of $.25, .45$, and $.95 \mathrm{sec}$ with suffix delays of $.25, .45$, and $.95 \mathrm{sec}$. For this experiment he used computer-generated speech, which insured accuracy of timing and absolute identity of the suffix under all conditions. The theory predicts that the suffix changes the shape of the serial position curve, so an appropriate metric is the proportion of the total errors which are made on the final item or items; in this way the effects of the suffix are distinguished from those due to factors which affect errors on all serial positions. Using this measure, Crowder showed that the size of the suffix effect was a monotonic function of the delay for the two fastest presentation rates. Crucially, for the hypotheses under test, at an interitem rate of $45 \mathrm{sec}$, the suffix at $.25 \mathrm{sec}$ after the last item had more effect than a suffix at $.45 \mathrm{sec}$. This result would favor the time hypothesis over the rhythm hypothesis. At a presentation rate of $.95 \mathrm{sec}$, however, the $.25-\mathrm{sec}$ delay suffix had less effect than the $.45-\mathrm{sec}$ suffix; and the .95 -sec suffix, although producing less effect than the .45 -sec suffix, was roughly equivalent to the .25 -sec suffix (Crowder, 1971, Figure 6b). Since 
the analysis of variance of this data showed only a significant main effect of suffix delay and no significant interaction, any detailed interpretations of the data must be treated with great circumspection. However, it seems fair to conclude that at the $.95 \mathrm{-sec}$ rate it is not really possible to exclude either of the two hypotheses. Indeed, there is no reason to reject the possibility that both factors are operating at the same time.

The present set of experiments, although they were carried out before Crowder's data were available, can be seen as a replication of his slowest rate of presentation. In addition, it is useful to have a replication with natural speech of experiments performed using synthetic speech. Crowder notes that his subjects received some training in listening to synthetic speech and that performance in a brief intelligibility test "indicated virtually perfect performance" (p. 333) Since Rabbitt (1968) has shown that memory tests are more sensitive than intelligibility tests to slight degradation of the stimulus items, it is possible that the nature of Crowder's stimuli introduced special factors.

\section{EXPERIMENT I}

\section{Method}

Design. Subjects from the APU subject panel were presented with lists of seven digits which had been recorded at a rate of one digit/sec. There were two crucial conditions in which a suffix followed the digit string at intervals including .5 and $1 \mathrm{sec}$. By the time hypothesis, the .5-sec suffix should give the greater effect; by the rhythm hypothesis, the 1-sec suffix should have more effect. In the test trials for each condition, the digits (from the set one to nine) were balanced for serial position. No digit was repeated in the same serial position in successive lists and no digit was repeated within a list. The digits and suffix were presented over a loudspeaker. A new series of digits began every $20 \mathrm{sec}$.

Subjects were instructed to begin recall as soon as they heard the last digit and to ignore the "nought." Subjects were asked to write the digits in the order they had heard them on prepared response sheets, writing from left to right, and to guess if unsure of any digit rather than leave blanks. A response was scored correct if the digit was both correct and in the correct serial position.

There were four conditions. One condition was a control, where there was no suffix after the digits. The other three conditions had a "nought" suffix coming after the last digit at one of three intervals: $.5,1$, and $1.5 \mathrm{sec}$. The materials were prerecorded and the suffixes were added at a second recording session. There were no apparent problems of timing of the suffixes.

Sixteen subjects from the APU subject panel were tested in four groups. Subjects were presented with 80 trials of seven-digit series in four blocks of 20 trials each, one block for each condition, the first two trials being unscored practice trials. The blocks were balanced across subjects for order of presentation.

Sixteen practice trials were given at the beginning of the session, four in each condition. Before each of the main blocks, subjects were told which condition they would hear. The first two items on each main block were not scored.

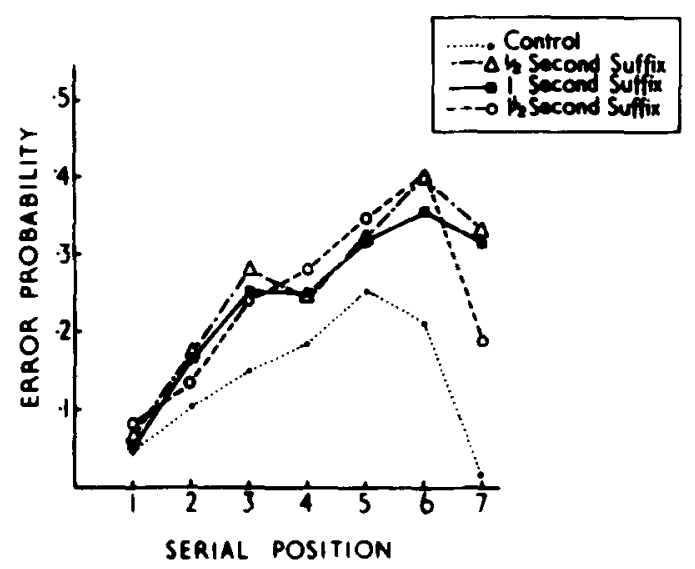

Figure 1. Error probabilities by serial position for suffixes played after the last digit at one of three intervals (Experiment I).

\section{Results}

The data are shown in Figure 1. There were no significant differences on the Wilcoxon test between the 1- and .5-sec suffixes. The 1.5-sec suffix had significantly fewer errors than the $.5-\sec (\mathrm{p}<.01)$ and the $1-\sec (p<.02)$ suffix on the last serial position. All three suffix conditions had significantly $(\mathrm{p}<.01$, Wilcoxon test) more errors on the last serial position than in the control condition. The differences between the $1.5-\mathrm{sec}$ suffix and the other two would be predicted by the time hypothesis. It is debatable whether it would be predicted by the rhythm hypothesis. The virtual identity between the .5- and 1-sec suffixes would not be predicted by either hypothesis. As we had expected the .5 -sec suffix to have the greater effect, both for theoretical reasons and on the basis of comments by the subjects (to have a 1-sec rhythm broken by a $.5-\mathrm{sec}$ interval is quite "disturbing"), we decided to repeat the first experiment using a new recording of the digits and a more mechanical method of recording the suffixes. The method used in Experiment I did not guarantee constant voice quality in the various conditions, a factor shown by Morton et al. (1971) to change the effect of the suffix.

\section{EXPERIMENT II}

This experiment involved a simple comparison between the .5- and 1-sec suffixes. In order to guarantee objective equivalence in voice quality, the suffix "ah" was recorded on a tape loop in a continuous form. A segment of the tape loop was gated electronically at the appropriate time and recorded onto the stimulus tape. Two groups of eight subjects listened to four blocks of 20 digit lists. The experiment started with a block of eight practice lists and the test blocks all began with two practice lists with the appropriate 


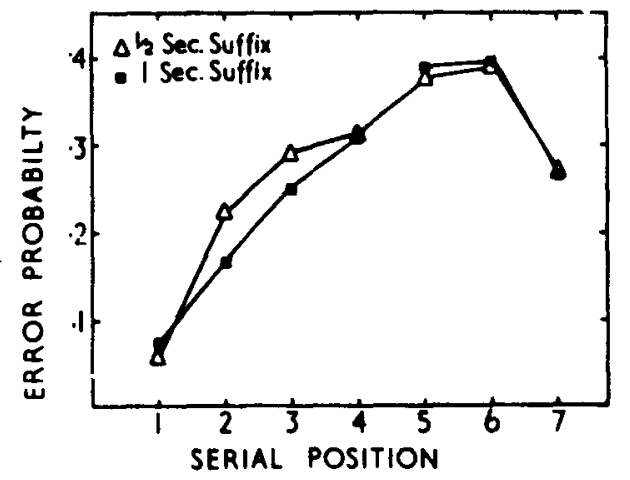

Figure 2. Error probabilities by serial position of .5- and $1-s e c$ interval suffixes (Experiment II).

suffix. Two of the test blocks had suffixes $.5 \mathrm{sec}$ after the final digit and two had a 1-sec suffix. One of the subject groups heard the suffix conditions in the order $.5,1,1, .5$ and the other $1, .5, .5,1$.

\section{Results}

The mean error probabilities are plotted in Figure 2. Wilcoxon tests showed no differences between the conditions except at Serial Position 2, where the .5-sec suffix condition was worse ( $p<.01$, two-tailed). As this result has no equivalent in the other experiment and has neither a coherent explanation nor any visible implications, we regard it as being due to chance variations.

\section{Conclusions}

In Experiment II, where the suffixes in the .5- and $1-\sec$ conditions were identical, there were no differences in recall which could be attributed to the timing of the suffix. This agrees with the result of Experiment I. Thus, we are forced to conclude that the size of the suffix effect is influenced both by the absolute timing of the suffix and its rhythm, i.e., its timing in relation to the timing of the items in the stimulus list. Effects due to the absolute timing of the suffix would be greater in the .5 -sec conditions and effects due to rhythm would be greater in the $1-\mathrm{sec}$ condition. In both Experiments I and II, these two influences are roughly equivalent.

It is, however, difficult to see how the two timing effects could be attributed to the same mechanism. The effect due to the proximity of the suffix to the last digit is most easily accounted for in terms of a relatively passive system, where the suffix is thought to overwrite the traces left behind in PAS. This is the account of suffix effect either implicitly or explicitly assumed in the earlier work on the suffix (Crowder, 1969, 1971; Crowder \& Morton, 1969; Morton, 1970; Morton et al., 1971). The effects of rhythm do not readily fit into such a mechanism, but rather seem to fit in with a more active account of short-term verbal memory such as that put forward by Neisser, Hoenig, and Goldstein (1969). These authors examined the stimulus prefix effect. The stimulus prefix is a redundant item presented immediately prior to a stimulus list but which does not have to be recalled. Its effect is to increase the number of errors made in recall equally on all serial positions (and as such is different from the suffix effect). Neisser et al. suggested that the prefix exerts its influence by interfering with the rhythmic organization which the subject imposes on the list to be remembered. They predicted that, if the prefix were made distinct, it would not be incorporated into the organization mechanism and thus would not affect recall. They tested this suggestion by presenting the prefix in a different voice from the stimulus list and also by presenting a group of three prefixes (the word "zero"). In the latter case, the triad of similar stimuli should form a single unit and, as such, should not affect the organization of the subsequent digits. Their predictions were successful. In the two test conditions, recall was as effective as in the condition where no prefix was present.

We can now look at the suffix effect in the same light and inquire how the effects of time and rhythm can coexist. The account of the suffix effect which I currently favor is as follows. During the presentation of a list of items, acoustic or visual, the material is organized in some way following categorization. With serial recall, a number of factors operate to impair recall of later items compared with earlier items. These factors will include aspects of the method of organization and retroactive interference of earlier responses on the recall of later items. With acoustic presentation, there is an additional source of information which is made available as a result of the properties of some part of the system which is responsible for analyzing speech inputs. This part of the system, called PAS, appears to store the final item for a little time. I suppose that some operation copies this information from PAS into another store in such a way that it is subject neither to input nor output interference. I further suppose that this operation is relatively autonomous. Any speech input arriving before this operation is complete (i.e., a suffix) has at least part of its effect by overwriting the information remaining in PAS. The extent of this effect would be a monotonic function of the time between the last item and the suffix. However, we can also suppose that the suffix can interfere with the storage operation which is responsible for preserving PAS information in the other store. There are a number of ways in which this might be done, but we could expect that they have in common the likelihood that the more the suffix appeared to be part of the stimulus list, in terms other than its semantic properties (Morton et al., 1971), the more it would interfere with the storage operation. This effect would correlate with the extent to which a suffix would overwrite PAS information but is seen as separate from it, since very large perceptual differences between stimuli 
and suffix (such as voice or ear of presentation) still result in appreciable suffix effects.

To test this idea, we performed the following two experiments. In both cases the interference with PAS information should be the same in all the conditions. The variable we examined was the influence of events occurring after the suffix on the size of the suffix effect.

\section{EXPERIMENT III}

If the suffix effect was entirely due to the overwriting of information in PAS which related to the final item in the list, then there should be no effect of any event which follows the suffix. We tested this by presenting the subjects with lists of digits followed either by a single suffix item ("ah") or by a group of either two or three identical suffixes. Nine examples of each of these suffix conditions were presented in random order together with a no-suffix condition. The stimuli were lists of eight digits. Each digit occurred equally often in all serial positions for all conditions. The digit lists were recorded in one session. At a second session, the lists were rerecorded onto one channel of a two-channel recorder. The experimenter monitored the lists through headphones and spoke the appropriate number of "ahs" at the end of the list.

The stimuli were presented to a group of 18 subjects in two blocks of 20 lists, the first two of which were not scored. The test trials were preceded by a block of 12 practice items. The digits and suffixes were presented at $.5-\mathrm{sec}$ intervals. Subjects were instructed to wait for the last suffix (if any) before starting to write their responses. Subjects had no apparent difficulty with the recall instructions.

\section{Results}

The mean error scores are plotted in Figure 3. All three suffix conditions give rise to a large suffix effect when compared with the control condition $(\mathrm{p}<.01$, two-tailed, at Serial Positions 6, 7, and 8 and total errors). Thus, unlike the Neisser et al. findings with the stimulus prefix, the effect of a stimulus suffix is not removed when the interfering item is repeated. Comparing the three suffix conditions, we find that the three-suffix condition gives rise to slightly fewer errors on the final serial position than does the single suffix $(p<.05)$. However, the three-suffix condition gives rise to more errors on Position 7 than either the oneor two-suffix conditions $(\mathrm{p}<.05)$, and more errors than the one-suffix condition at Position $4(\mathrm{p}<.01)$. In addition, the two-suffix condition is worse than the one-suffix condition at Position $4(\mathrm{p}<.02)$. Thus, the gains of the three-suffix condition at the final position are lost earlier in the list. This means that when the conditions are compared by the proportion of errors made on the final item (a measure used by Crowder, 1971), the difference between the three- and one-suffix conditions is greater $(\mathrm{p}<.01)$.

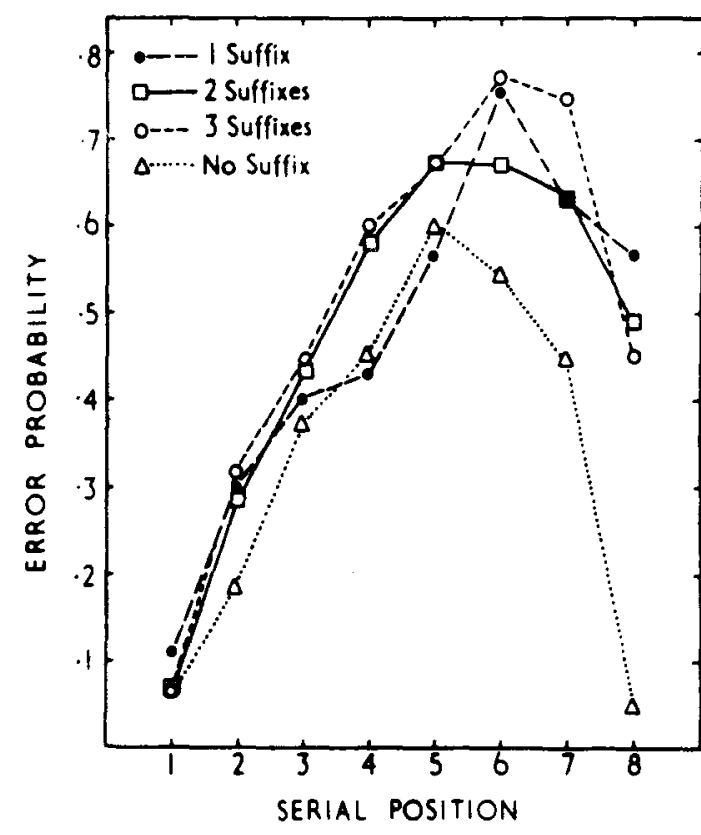

Figure 3. Error probabilities by serial position for a variety of suffixes including groups of either two or three identical suffixes (Experiment III).

\section{EXPERIMENT IV}

It was suggested by a reviewer with a delicate nose that the method used to record the suffixes might resuit in differences in voice quality in the case of the threesuffix condition. By this time we had developed a system for using a computer to record our stimulus lists and decided to repeat the previous experiment.

\section{Method}

Prototypical digits were recorded on magnetic tape and then transferred to a disk store via a Modular One computer system, using a sampling rate of $20 \mathrm{KHz}$ and an accuracy of 8 bits. A prototypical "ah" was similarly recorded. A further computer program was used to record the stimulus tapes by calling the appropriate items from disk into the store and outputting them through a DAC to a tape recorder. In this way, the identity of the stimuli and suffixes was maintained in the experimental conditions without the need to use synthetic speech.

Design. There were three conditions of 18 trials each: a single-suffix, a triple-suffix condition, and a no-suffix condition. Each trial consisted of eight digits presented at one every $.65 \mathrm{sec}$. The suffixes followed at a similar rate. The three conditions were randomized and preceded by a block of 18 practice lists.

Subjects. A single group of 16 female volunteer subjects was used. The procedure followed that of the previous experiment.

\section{Results}

The error curves are shown in Figure 4. In the crucial comparison between the two experimental conditions on the final serial position, there were significantly fewer errors in the three-suffix conditions than in the one-suffix condition $(p<.05)$. This replication of a weak effect gives us more confidence to accept it as genuine. 


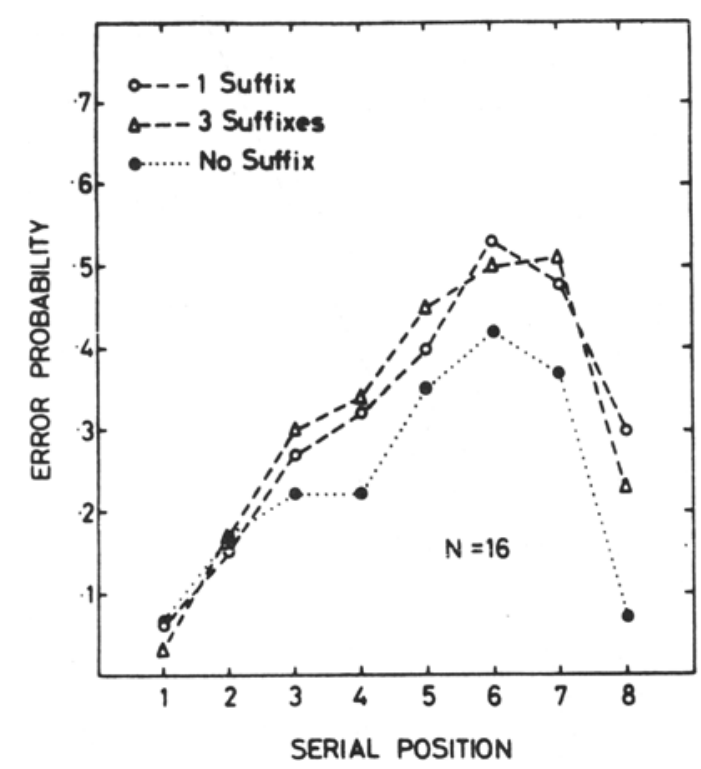

Figure 4. Error probabilities by serial position comparing a single suffix with a group of three suffixes: stimulus lists produced with computer aid (Experiment IV).

\section{EXPERIMENT V}

One aspect of the suffixes previously used which might cause them to be grouped with the stimulus items is that they have normal speech durations. In this experiment we investigated the effect of a prolonged suffix, an "ah" vocalization prolonged for $2 \mathrm{sec}$. Two such suffixes were used, one with a normal onset of vocalization $.5 \mathrm{sec}$ after the final stimulus item and the other which was started about $2 \mathrm{sec}$ before the final item and continued for about $4.5 \mathrm{sec}$. This suffix was initially recorded on the second channel of a twintrack machine and was electronically gated onto the final stimulus tape so that it started $.5 \mathrm{sec}$ after the final stimulus item. The two prolonged suffixes then differed only in the onset characteristics. These suffixes were compared with a normal "ah" suffix and a control condition with no suffix in a design identical to the one used in Experiment III. The stimuli were presented to a group of 13 members of the APU subject panel.

\section{Results}

The mean error scores are plotted in Figure 5. Since there were no differences between the two prolonged suffixes, the scores on those two conditions have been combined. The usual suffix effects are apparent with both kinds of suffixes. The prolonged suffixes, however, gave rise to significantly fewer errors than the normal suffix on the final serial position $(p<.01$, Wilcoxon, two-tailed). On the penultimate position, this difference was reversed, with the prolonged suffixes causing more errors $(\mathrm{p}<.01)$. However, the reviewer previously mentioned suggested that voice quality might not be the same with a long vowel as with a short vowel and that small differences in voice quality could give rise to the difference found. So, we decided to repeat the experiment using the computer system described under Experiment IV.

\section{EXPERIMENT VI}

Three conditions were used. In one, the suffix was a normal "ah" which was slightly truncated at the end to remove the normal fall characteristics. This procedure has been shown to have no effect on the size of the suffix effect (Morton \& Chambers, in press). The second suffix started off in exactly the same way but was processed in the computer, so that the final pitch pulse was repeated to bring the total time for the suffix to 4.5 sec. This grafting was accomplished without any sharp transition. The final part of the vowel sounded slightly unusual, but that, if anything, should favor a reduction in the suffix effect (Morton \& Chambers, in press); the vital point is that the first $300 \mathrm{msec}$ of these suffixes were identical. The third suffix was a $41 / 2$-sec buzz rather than a silent control. to confirm that a long suffix had no secondary effects by, for example, delaying the subject's response.

The rest of the design and method followed Experiment IV. The subjects were 25 members of the APU subject panel, tested in two groups.

\section{Results}

The results are shown in Figure 6. There are no differences between the two "ah" suffixes, which differed significantly from the control on the final serial positions. We conclude that the results of

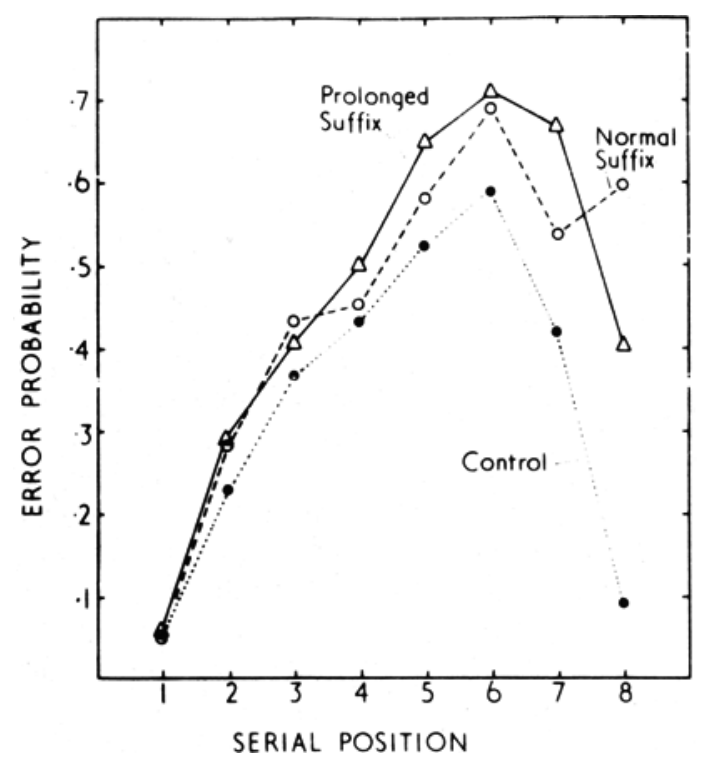

Figure 5. Error probabilities by serial position for prolonged and normal suffixes (Experiment V). This result is considered to be artifactual (see text). 
Figure 6. Error probabilities by serial position for prolonged and normal suffixes: stimulus lists produced with computer aid (Experiment VI). This null result is considered to negate that of Experiment $V$.

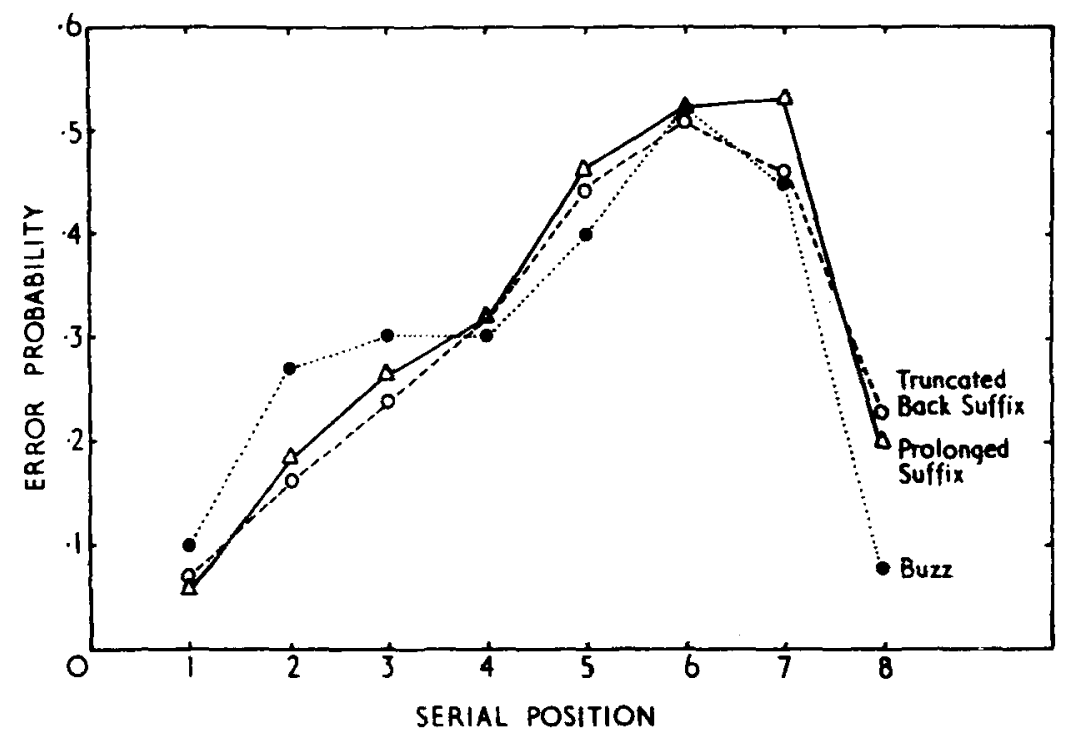

Experiment $\mathrm{V}$ were indeed the consequence of changes in voice quality; we report them still as a methodological caution.

\section{DISCUSSION}

The results of Experiments III and IV seem to bear out the earlier suggestion of two separate operations in the suffix effect. The manipulation of repeating the suffix should have had no effect on the purely passive, mechanical overwriting of information which constituted the earlier account of the suffix effect. The fact that the manipulation did not remove the suffix effect is evidence that the passive system plays the major role. The reduction in the suffix effect, however, indicates that some other mechanism is involved as well. It was suggested above that the process responsible for copying PAS information into an interference-free store could account for the effects of rhythm. It could also account for the reduced effect found in Experiments III and IV. The precise operation of this mechanism is obscure. Whatever account is finally acceptable, it is now clear that the effect of the suffix and, accordingly, the difference between visual and acoustic presentation with serial recall do not have a simple, single explanation.

\section{REFERENCES}

Crowder, R. G. Improved recall for digits with delayed cues. Journal of Experimental Psychology, 1969, 82, 258-262.

Crowder. R. G. Waiting for the stimulus suffix: Decay, delay. rhythm, and readout in immediate memory. Quarterly Journal of Experimental Psychology, 1971, 23, 324-340.

Crowder. R. G.. \& Morton. J. Precategorical acoustic storage (PAS). Perception \& Psychophysics, 1969. 5. 305-373.

Morton. J. A functional model for memory. In D. A. Norman (Ed.). Models of human memory. New York: Academic Press. 1970. Pp. 203-254.

Morton, J.. Crowder, R. G.. \& Prussin, H. A. Experiments with the stimulus suffix effect. Journal of Experimental Psychology Monogruph, 1971, 91, 169-190.

Morton. J.. \& Chambers. S. M. Some evidence concerning speech as an acoustic feature. British Journal of Psychology. in press.

Neisser. U.. Hoenig. Y. J.. \& Goldstein. E. Perceptual organization in the prefix effect. Journal of Verbal Learning and Verbal Behavior. 1969. 8. 424-429.

RABBitT, P. M. A. Channel capacity. intelligibility and immediate memory, Quanerly Journal of Experimental Psychology. 1968, 20. $241-248$.

Routh. D. A. Independence of the modality effect and amount of silent rehearsal in immediate memory. Journal of Verbal Leaming and Verbal Behavior. 1971, 10. 213-218.

(Received for publication May 8, 1974; revision received May 14, 1975; accepted June 9,1975 .) 